\title{
Pulmonary venous flow velocity patterns in 404 individuals without cardiovascular disease
}

\author{
S F de Marchi, M Bodenmüller, D L Lai, C Seiler
}

\begin{abstract}
Objective-To determine the pulmonary venous flow velocity (PVFV) values in a large normal population.

Design-Prospective study in consecutive individuals.

Setting-University hospital.

Methods-Among 404 normal individuals, the flow velocity pattern in the right upper pulmonary vein was recorded in 315 subjects using transthoracic echocardiography, and in both upper pulmonary veins in 100 subjects using transoesophageal echocardiography. Subjects were divided into five age groups. The PVFV values were compared between transthoracic and transoesophageal echocardiography within the age groups, and intraindividually between the right and left upper pulmonary veins in transoesophageal echocardiography.

Results-Normal PVFV values for the right upper pulmonary vein in transthoracic and transoesophageal echocardiography are presented. The duration of flow reversal at atrial contraction was overestimated using transthoracic echocardiography (mean (SD): 96 (21) ms in transoesophageal echocardiography, 120 (28) $\mathrm{ms}$ in transthoracic echocardiography, $\mathrm{p}<0.0001)$. Systolic to diastolic peak flow velocity ratio (S:D) increased earlier with advancing age with transoesophageal echocardiography than with transthoracic echocardiography. Similar results were found for the corresponding time-velocity integrals. Data from the left and right upper pulmonary veins differed with respect to onset and deceleration of flow velocities, but not for flow durations or peak velocities.
\end{abstract}

Conclusions-Normal PVFV values generally show a wide range. The data presented will be of value in assessing left ventricular diastolic function and mitral regurgitation using the PVFV pattern.

(Heart 2001;85:23-29)

Keywords: pulmonary venous flow velocity; Doppler echocardiography; mitral regurgitation

Increasing attention is being paid to the Doppler pulmonary venous flow velocity (PVFV) pattern, because it has been shown to provide clinically important information on ventricular and valvar function. In particular, it has been shown to be a specific indicator of severe mitral regurgitation. ${ }^{1-3}$ In addition, promising results have been obtained in the non-invasive assessment of left ventricular loading conditions using PVFV in addition to the left ventricular inflow pattern. ${ }^{4}$ Several new approaches - such as myocardial or mitral annular motion velocity measurements using Doppler tissue imaging and colour $M$ mode recordings of left ventricular inflow-have been developed to assess left ventricular relaxation independently of loading conditions. ${ }^{6-8}$ However, PVFV measurement as an adjunct to transmitral flow velocity assessment remains the most accessible and widely used method in routine Doppler echocardiography for characterising mitral regurgitation and left ventricular diastolic function.

In a study by Gentile and colleagues, the PVFV patterns were determined in 143 normal subjects by transthoracic echocardiography. ${ }^{9}$ The number of individuals, however, was too small to establish accurate normal values according to age classes. Our aim in the present study was therefore to determine PVFV parameters in a large normal population, thus allowing a classification into different age groups. In addition, the PVFV patterns were compared between transthoracic and transoesophageal echocardiography, as well as between the right and left upper pulmonary veins during transoesophageal echocardiography.

\section{Methods}

STUDY POPULATION, INCLUSION CRITERIA In all, 404 consecutive individuals undergoing routine clinical echocardiographic evaluation in our hospital were included prospectively. The Doppler flow velocities of the right upper pulmonary vein were assessed in 315 of these using transthoracic echocardiography, and in 100 using transoesophageal echocardiography. In 11 subjects, both transthoracic and transoesophageal echocardiography were performed. Thus the total number of right upper pulmonary vein assessments by Doppler echocardiography was 415 . In 72 subjects undergoing transoesophageal echocardiography, flow velocities in the left upper pulmonary vein were also assessed.

All subjects were included on the basis of the following criteria:

- no history of cardiovascular disease or hypertension;

- no evidence of cardiovascular disease on physical examination;

- no cardiovascular drugs being taken;

- normal ECG;

- normal echocardiography.
Accepted 12 September 2000 
A

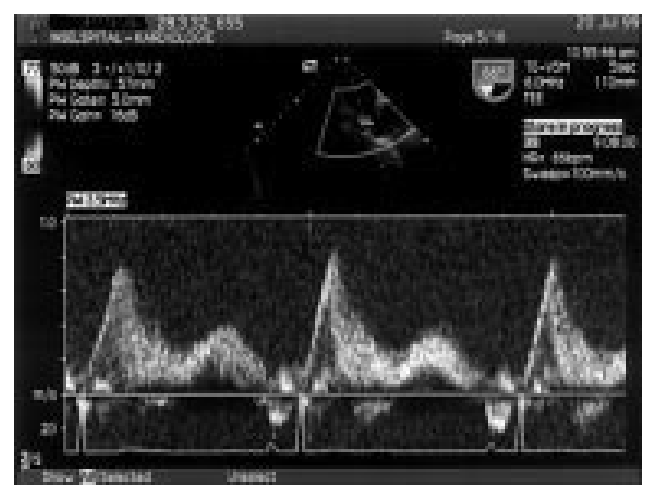

B

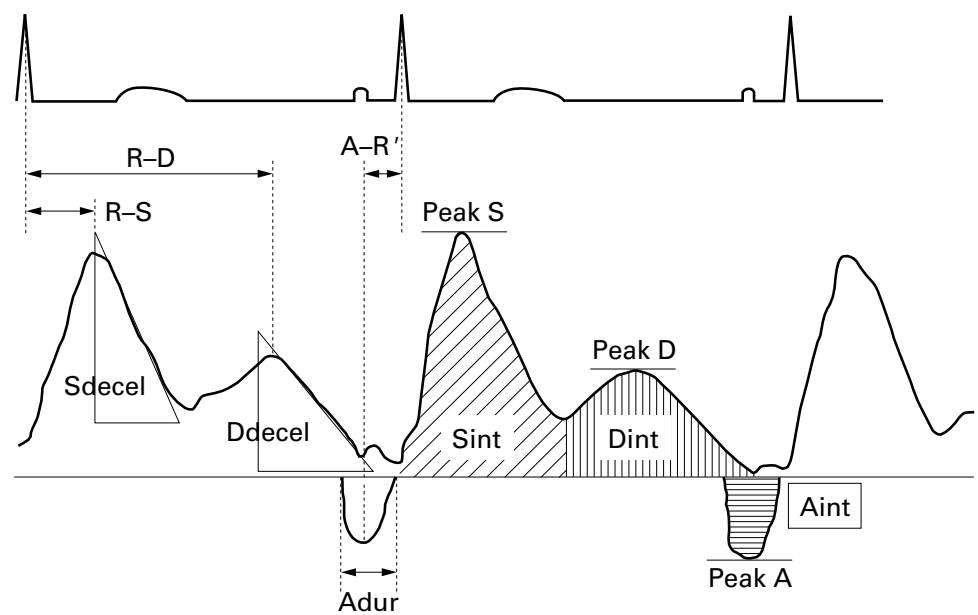

Figure 1 (A) Transoesophageal Doppler echocardiographic recording of right upper pulmonary venous flow in a 67 year old man. (B) Illustration of the flow velocity variables measured in each pulmonary vein. Explanations of abbreviations are given in the box.

The echocardiographic study was considered normal if the following criteria were fulfilled:

- normal global and regional systolic ventricular function;

- absence of more than mild valvar regurgitation;

- absence of more than mild valvar stenosis;

- normal transmitral inflow pattern, consistent with normal left ventricular diastolic function;

- absence of detectable intracardiac or extracardiac shunts, except for a patent foramen ovale;

- absence of ventricular hypertrophy;

- absence of atrial, ventricular, or vessel dilatation.

TRANSTHORACIC ECHOCARDIOGRAPHY

All transthoracic echocardiographic studies were performed on an Acuson Sequoia, Acuson Aspen, or Acuson XP128 ultrasound system (Acuson, Mountainview, California, USA). All ultrasonography systems were equipped with $2-5 \mathrm{MHz}$ transducers, as well as continuous wave and pulsed wave Doppler and colour Doppler imaging. Subjects were examined in the left lateral supine position. All echocardiographic examinations were recorded on VHS videotape for off-line analysis.

$M$ mode and cross sectional echocardiography End systolic left atrial diameter and the end diastolic diameter of the ascending aorta were measured using $M$ mode echocardiography from a parasternal view. Left ventricular end diastolic diameter and the end systolic and end diastolic thickness of the septal and posterior wall were measured. Left ventricular mass index was calculated using $M$ mode echocardiography, according to the recommendations of the American Society of Echocardiography. ${ }^{10}$ From the apical four and two chamber views, cine clips of two dimensional scans of the left ventricle were acquired for the calculation of left ventricular ejection fraction according to the area-length method.
Doppler echocardiography

Transmitral flow was assessed from the apical four chamber view. The pulsed wave sample volume was placed at the tips of the mitral leaflets. The variables obtained included: peak velocity (peak E, $\mathrm{cm} / \mathrm{s}$ ) and deceleration time of early filling (E-DT, ms), peak velocity (peak A, $\mathrm{cm} / \mathrm{s}$ ) and duration (Adur $\mathrm{TMF}_{\mathrm{TMF}}, \mathrm{ms}$ ) of late (atrial) filling, early to late diastolic peak velocity ratio (E:A), time-velocity integral of the entire diastolic transmitral flow (TMFint), and isovolumetric relaxation time (ms).

Pulmonary venous flow velocities were assessed from the apical four chamber view. The pulsed wave Doppler sample volume was placed $1 \mathrm{~cm}$ upstream in the right upper pulmonary vein. Sample volume positioning was always guided by colour Doppler imaging. The variables obtained are shown in fig 1 and listed in the box below. The difference between the duration of the pulmonary venous flow reversal at atrial contraction and the transmitral forward flow at atrial contraction (A - Adur $_{\mathrm{TMF}}, \mathrm{ms}$ ) was calculated.

\section{TRANSOESOPHAGEAL ECHOCARDIOGRAPHY}

For transoesophageal echocardiography, the same ultrasound equipment was used as for transthoracic echocardiography. The frequency of the omniplane transducer ranged from 2-7.5 MHz. The Doppler facilities used in transthoracic echocardiography were also available for transoesophageal echocardiography. Before insertion of the transoesophageal probe, $2-3 \mathrm{mg}$ of midazolam were given intravenously for sedation. A three lead ECG, respiratory changes, systemic blood pressure, and blood oxygen saturation were recorded continuously. Measurements were performed with the subjects in the left lateral supine position. All echocardiographic examinations were recorded on VHS videotape for off-line analysis.

\section{Doppler echocardiography}

Transmitral flow velocities were measured using pulsed wave Doppler echocardiography in the transverse plane. The pulmonary venous 
Doppler flow patterns in the right and left upper pulmonary veins were obtained in the plane giving the best visualisation of the flow velocity signals.

DATA ANALYSIS

Classification of study subjects

Subjects were classified according to their decade of life. The lowest age group included individuals 10-19 years old, the highest group individuals $\geqslant 60$ years old.

Analysis of PVFV variables

The Doppler flow velocity patterns of the right upper pulmonary vein were compared within the different age groups between 315 subjects undergoing transthoracic echocardiography and 100 subjects undergoing transoesophageal echocardiography. Age dependency of the various PVFV indices was assessed using linear regression analysis. For non-age-dependent variables, the corresponding normal values were calculated for all subjects and compared between transthoracic and transoesophageal echocardiography. In 72 individuals undergoing transoesophageal echocardiography, the pulmonary venous Doppler flow pattern was compared intraindividually between right and left upper pulmonary veins.

\section{Assessment of reproducibility and feasibility of measurements}

In a subset of 110 randomly selected individuals, a second independent investigator repeated all measurements from the Doppler videotapes in order to assess interindividual reproducibility. These measurements consisted of 50 transthoracic and 60 transoesophageal echocardiograms, the latter involving 30 from the right upper pulmonary vein and 30 from the left upper pulmonary vein.

In 100 additional consecutive patients referred for routine clinical transthoracic echocardiography and 25 patients referred for transoesophageal echocardiography, we assessed the feasibility of the PVFV measurements prospectively. In order to provide information on the feasibility of Doppler PVFV measurements in a clinical setting, patients in this additional study group were not selected on the basis of the presence or absence of cardiovascular disease but were included consecutively with-

\section{Abbreviations used for physiological} terms

Adur $_{\text {TMF }}$, peak duration (ms) of late (atrial) filling

Aint, time-velocity integral of reverse flow at atrial contraction (m)

A-R', time from peak A wave velocity to the following ECG $\mathrm{R}$ wave (ms)

$\mathrm{E}: \mathrm{A}$, early to late diastolic peak velocity ratio E-DT, deceleration time of early transmitral filling (ms)

Ddecel, deceleration time of antegrade diastolic flow (ms)

Dint, time-velocity integral of antegrade diastolic flow (m)

E:A, early to late diastolic transmitral peak flow velocity ratio

Peak A, peak reverse flow velocity at atrial contraction $(\mathrm{cm} / \mathrm{s})$

Peak D, peak antegrade diastolic flow velocity $(\mathrm{cm} / \mathrm{s})$

Peak E, peak velocity of early filling $(\mathrm{cm} / \mathrm{s})$

Peak S, peak systolic flow velocity $(\mathrm{cm} / \mathrm{s})$

$\mathrm{R}-\mathrm{S}$ time, time from ECG $\mathrm{R}$ wave to peak systolic flow velocity (ms)

$\mathrm{R}-\mathrm{D}$ time, time from ECG $\mathrm{R}$ wave to peak diastolic flow velocity (ms)

$\mathrm{S}: \mathrm{A}$, systolic to diastolic peak velocity flow ratio

Sdecel, deceleration time of systolic flow (ms)

Sint, time-velocity integral of systolic flow (m)

TMFint, time-velocity integral of the entire diastolic transmitral flow (m)

out any exclusion criteria. We documented our ability to obtain systolic, diastolic, and reversed flow signals in each patient. Feasibility was calculated as the percentage of patients in whom a Doppler flow signal was obtainable.

\section{Descriptive and comparative statistics}

The PVFV variables obtained were expressed as mean (SD) within each study group. For comparisons of normal values between transoesophageal and transthoracic echocardiography within age groups, unpaired Student's $t$ tests were performed. For intraindividual comparisons of normal values between right and

Table 1 Demographic and echocardiographic characteristics

\begin{tabular}{|c|c|c|c|c|c|c|c|}
\hline & $10-19$ years & 20-29 years & $30-39$ years & $40-49$ years & $50-59$ years & $\geqslant 60$ years & All subjects \\
\hline $\mathrm{n}$ & 15 & 92 & 127 & 65 & 52 & 50 & 404 \\
\hline Age (years) & $17.9(1.0)$ & $25.1(3.0)$ & $34.4(2.9)$ & $44.4(3.0)$ & $54.1(3.0)$ & $69.2(7.3)$ & $40.3(15.0)$ \\
\hline Male/female (\%male) & $15 / 3(80 \%)$ & $63 / 29(68 \%)$ & $93 / 34(73 \%)$ & $33 / 32(51 \%)$ & $29 / 23(56 \%)$ & $27 / 23(54 \%)$ & $259 / 145(64 \%)$ \\
\hline LVMI $\left(\mathrm{g} / \mathrm{m}^{2}\right)$ & $75(9)$ & $85(25)$ & $87(22)$ & $86(15)$ & $88(19)$ & $90(21)$ & $88(22)$ \\
\hline LA diameter (mm) & $34(4)$ & $33(5)$ & $35(5)$ & $34(5)$ & $36(4)$ & $37(6)$ & $35(5)$ \\
\hline LVEF (\%) & $67(7)$ & $68(7)$ & $69(7)$ & $69(7)$ & $69(6)$ & $69(7)$ & $69(7)$ \\
\hline Heart rate (beats/min) & $74(9)$ & $70(14)$ & $68(13)$ & $75(17)$ & $73(13)$ & $72(12)$ & $71(14)$ \\
\hline Systolic BP (mm Hg) & $123(24)$ & $120(10)$ & $121(15)$ & $123(16)$ & $124(19)$ & $131(24)$ & $123(17)$ \\
\hline Diastolic BP (mm Hg) & $73(13)$ & $71(11)$ & $74(12)$ & $75(11)$ & $73(11)$ & $72(14)$ & $73(12)$ \\
\hline TMFint (m) & $0.191(0.030)$ & $0.184(0.035)$ & $0.194(0.046)$ & $0.191(0.050)$ & $0.172(0.031)$ & $0.198(0.040)$ & $0.189(0.042)$ \\
\hline $\mathrm{E}: \mathrm{A}$ & $1.61(0.37)$ & $1.81(0.52)$ & $1.52(0.43)$ & $1.31(0.32)$ & $1.12(0.27)$ & $0.98(0.22)$ & $1.49(0.48)$ \\
\hline EDT (ms) & $144(26)$ & $152(35)$ & $164(38)$ & $167(41)$ & $166(41)$ & $165(47)$ & $161(39)$ \\
\hline IVRT (ms) & $73(7)$ & $71(10)$ & $76(14)$ & $72(10)$ & $79(14)$ & $83(14)$ & $75(13)$ \\
\hline $\operatorname{Adur}_{\mathrm{TMF}}(\mathrm{ms})$ & $130(20)$ & $139(24)$ & $146(34)$ & $143(31)$ & $142(19)$ & $155(35)$ & $144(30)$ \\
\hline
\end{tabular}

Values are mean (SD)

Adur $_{\mathrm{TMF}}$, duration of late diastolic transmitral flow; BP, blood pressure; E:A, early to late diastolic peak flow velocity ratio; EDT, deceleration time of early transmitral filling; IVRT, isovolumetric relaxation time; LA, left atrial; LVEF, left ventricular ejection fraction; LVMI, left ventricular mass index; TMFint, time-velocity integral of transmitral flow. 


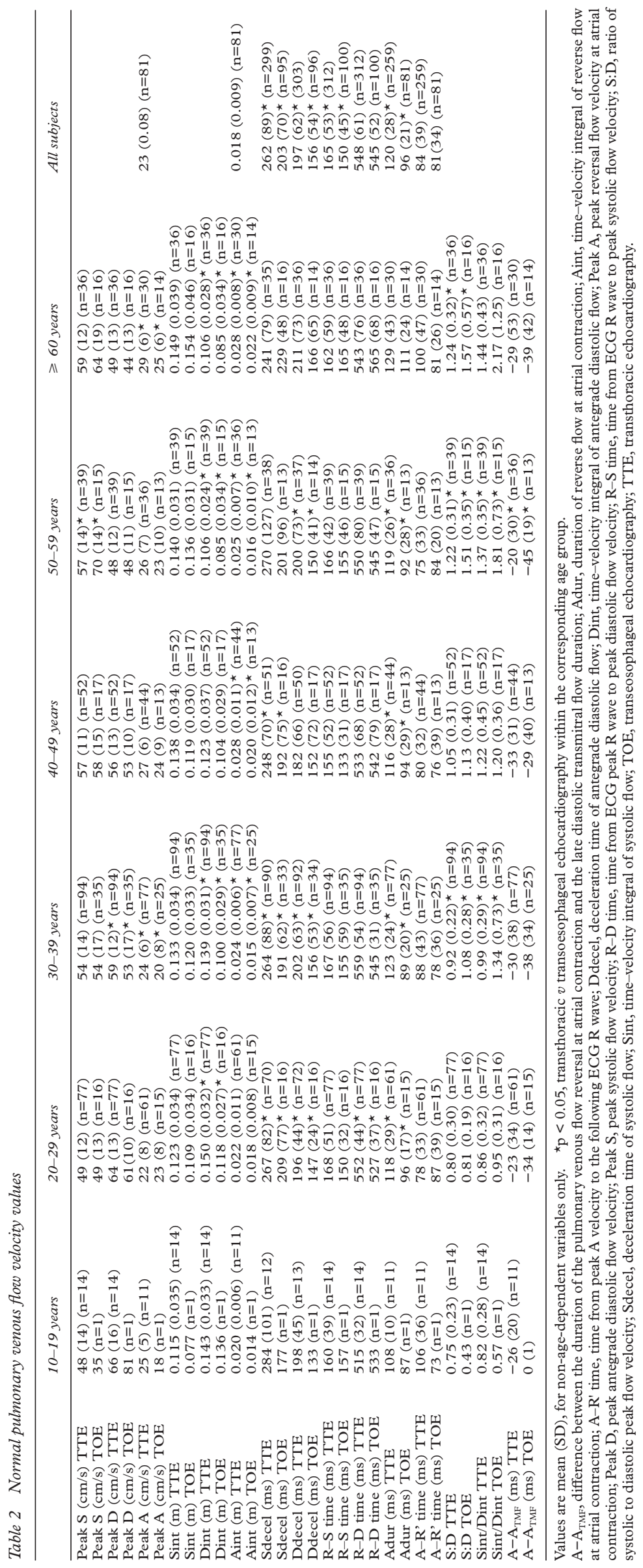

left upper pulmonary veins, we used paired Student's $t$ tests. Linear regression analysis was performed to determine the age dependency of the flow measurements. Interobserver variability was quantified using the mean difference and the standard error of estimate (SEE) of the two observer's values.

\section{Results}

BASELINE CHARACTERISTICS OF THE STUDY

INDIVIDUALS

The baseline characteristics of the study individuals are listed in table 1. All echocardiographic variables were within normal limits, reflecting the inclusion criteria. There was a predominance of male subjects in all age groups, ranging from $51-80 \%$. Left ventricular mass index, left atrial systolic diameter, systolic blood pressure, deceleration time of early transmitral filling, isovolumetric relaxation time, and the duration of late diastolic transmitral filling increased with age, whereas early to late diastolic transmitral peak velocity ratio decreased $(p<0.05$ for all linear regression analyses). Systolic blood pressure was significantly higher in transthoracic echocardiography than in transoesophageal echocardiography in the third and fifth decade group and in all subjects $(p<0.02)$. Diastolic blood pressure was higher in transthoracic echocardiography in the fifth and sixth decade group and in all subjects $(p<0.05)$. TMFint was higher in transthoracic echocardiography in all age groups $(p<0.05)$ except in the oldest group. Mitral valve Adur $_{\mathrm{TMF}}$ was longer in transthoracic echocardiography in the third and fourth decade group and in all subjects $(p<0.02)$. Isovolumetric relaxation time was shorter in transthoracic echocardiography in the third and in the sixth decade group and in all subjects $(p<0.05)$. Heart rate was lower in transthoracic echocardiography in the second, third, and fifth decade group and in all subjects $(p<0.02$, table 2$)$.

REPRODUCIBILITY AND FEASIBILITY OF PVFV MEASUREMENTS

For right upper pulmonary vein measurements, the ratio of systolic to diastolic peak flow velocity (S:D) was highly reproducible using both transthoracic echocardiography (mean (SEE) difference $=0.007(0.09)$ ) and transoesophageal echocardiography $\quad(0.02$ (0.25)). Peak A showed moderate interobserver variability: $0.1(3.9) \mathrm{cm} / \mathrm{s}$ for transthoracic echocardiography; $2.0(3.7) \mathrm{cm} / \mathrm{s}$ for transoesophageal echocardiography. Velocity measurements in general, including velocities from all measurements during the cardiac cycle, were highly reproducible in both transthoracic and transoesophageal echocardiography (fig 2). Adur, however, showed more pronounced variability (mean (SEE) difference $=2.9$ (15) ms for transthoracic echocardiography, 7.1 (14) ms for transoesophageal echocardiography). Left upper pulmonary vein measurements generally showed higher interobserver variabilities than the corresponding right upper pulmonary vein measurements (mean (SEE) 

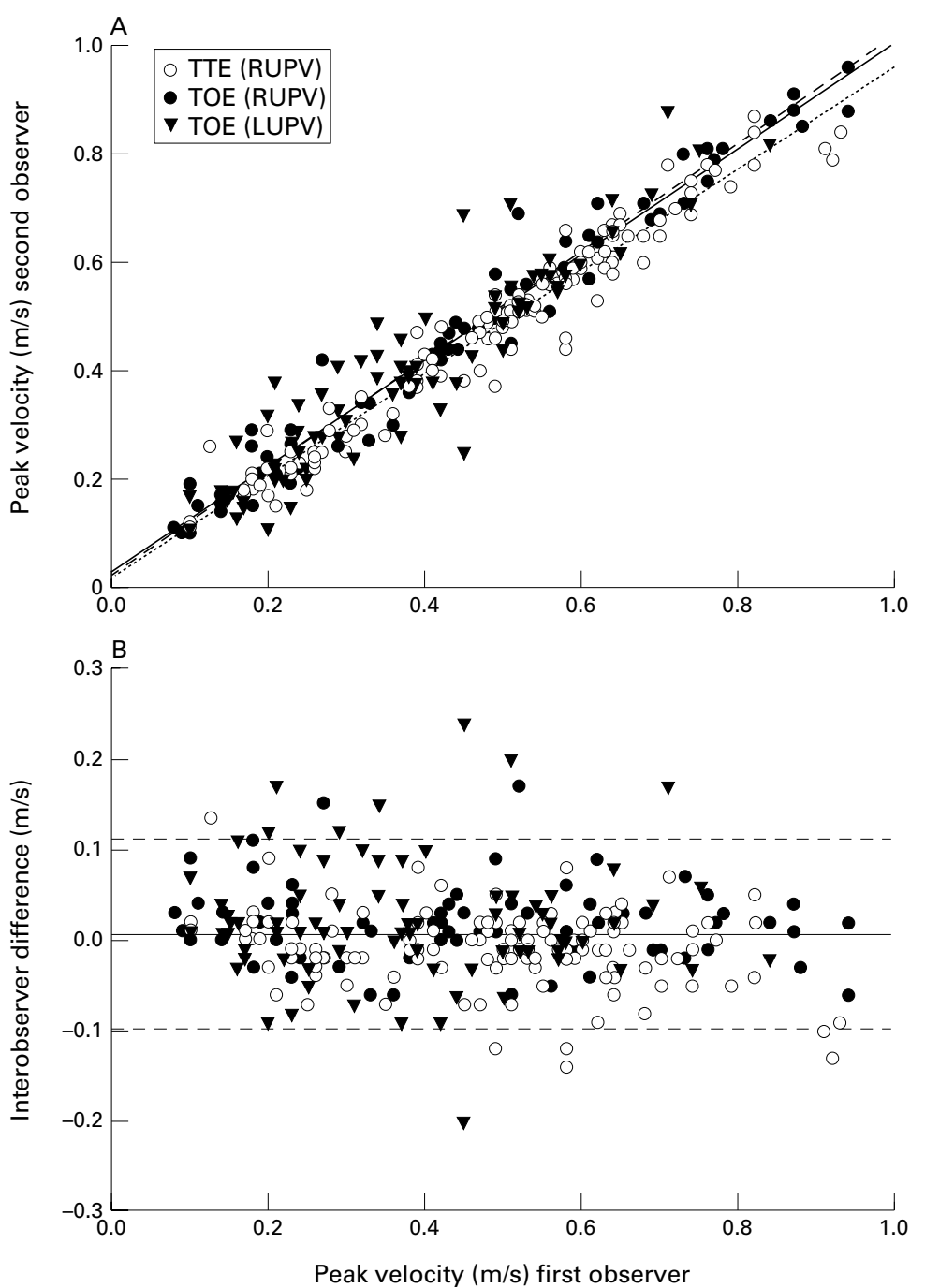

Figure 2 (A) Correlation between first and second observers'velocity measurements (systolic, diastolic, and reversed flow at atrial contraction) in transthoracic and transoesophageal echocardiography. (B) Bland-Altmann plot showing the interobserver variability of pulmonary venous flow velocity measurements in transthoracic and transoesophageal echocardiography. The solid line indicates the mean and the dashed lines the $95 \%$ confidence intervals of the interobserver difference of all flow velocity measurements. LUPV, left upper pulmonary vein; RUPV, right upper pulmonary vein; TOE, transoesophageal echocardiography; TTE, transthoracic echocardiography.

difference $=0.02(0.30)$ for $\mathrm{S}: \mathrm{D}$ ratio; 1.5 (6.1) $\mathrm{cm} / \mathrm{s}$ for peak A; 26 (29) $\mathrm{ms}$ for Adur).

In transthoracic echocardiography, systolic forward flow assessment was feasible in 96 of 100 patients, whereas diastolic forward flow was attainable in 94 and reversed flow at atrial contraction in 79 . In transoesophageal echocardiography, the feasibility of systolic, diastolic, and reversed flow assessment was $25 / 25$ (100\%), 25/25 (100\%), and 24/25 (96\%), respectively, for the right upper pulmonary vein, and 23/25 (92\%), 23/25 (92\%), and $19 / 25(76 \%)$ for the left upper pulmonary vein. These data are in agreement with a previous study by Jensen and colleagues. ${ }^{11}$

AGE DEPENDENCY OF PVFV VARIABLES: LINEAR REGRESSION ANALYSES

S:D was directly correlated with age in both transthoracic $(r=0.51, \mathrm{p}<0.0001)$ and transoesophageal echocardiography $(r=0.59$, $\mathrm{p}<0.0001$ for right upper pulmonary vein; $r=0.51, \mathrm{p}<0.0001$ for left upper pulmonary vein; fig 3). This increase with age reflected a significant increase in peak $\mathrm{S}$ in the right upper pulmonary vein $(r=0.27, \mathrm{p}<0.0001$ for transthoracic echocardiography and $r=0.40$, $\mathrm{p}=0.0001$ for transoesophageal echocardiography), as well as a decrease in peak $\mathrm{D}$ in both left upper pulmonary vein $(r=-0.52$, $\mathrm{p}<0.0001)$ and right upper pulmonary vein $(r=-0.40, \mathrm{p}<0.0001$ for transthoracic echocardiography and $r=-0.34, \mathrm{p}=0.001$ for transoesophageal echocardiography). Analogous results were found for the velocity-time integrals. There was a delayed S:D increase with increasing age in transthoracic compared with transoesophageal echocardiography. The regression line exceeded the value $S: D=1$ (dotted line in fig 3) at the age of 41 years in transthoracic echocardiography, and at the age of 32 years in transoesophageal echocardiography (dotted arrows in fig 3). Peak A and Aint were significantly related to age in transthoracic echocardiography $(r=0.31$, $\mathrm{p}<0.0001)$, but not in transoesophageal echocardiography. There was a trend for Adur to be directly related to age, but this was not significant $(r=0.12, \mathrm{p}=0.07$ for transthoracic echocardiography and $r=0.19, \mathrm{p}=0.08$ for transoesophageal echocardiography).

NORMAL VALUES FOR THE RIGHT UPPER PULMONARY VEIN ACCORDING TO THE DECADE OF LIFE

The normal values for the right upper pulmonary vein were classified into six age groups and are listed in table 2 for transthoracic and transoesophageal echocardiography separately. Compared with transoesophageal echocardiography, the values for transthoracic echocardiography showed a significantly lower peak $S$ in the fifth decade, a higher peak $\mathrm{A}$ in the third and sixth decade, and a longer Adur in the second and third decade. Adur tended to be shorter in all age groups in transoesophageal than in transthoracic echocardiography. PVFV variables that were not found to be age dependent in linear regression analysis were averaged for all subjects. Mean values of deceleration time of systolic flow (Sdecel), deceleration time of antegrade diastolic flow (Ddecel), time from ECG $\mathrm{R}$ wave to peak systolic flow velocity (R-S time), and Adur were found to be significantly longer in transthoracic than in transoesophageal echocardiography. In particular, mean (SD) Adur was 120 (28) $\mathrm{ms}$ in transthoracic echocardiography and 96 (21) ms in transoesophageal echocardiography $(\mathrm{p}<0.0001)$.

NORMAL VALUES FOR THE RIGHT AND LEFT UPPER PULMONARY VEINS: INTRAINDIVIDUAL COMPARISONS

As comparisons between right and left upper pulmonary veins were performed intraindividually, data were not split into different age groups, but are displayed as mean (SD) for the entire group of 72 individuals (table 3 ). Sdecel and $\mathrm{R}-\mathrm{S}$ time were significantly shorter and $\mathrm{R}-\mathrm{D}$ time longer in the right upper pulmonary vein. No significant differences were found in 


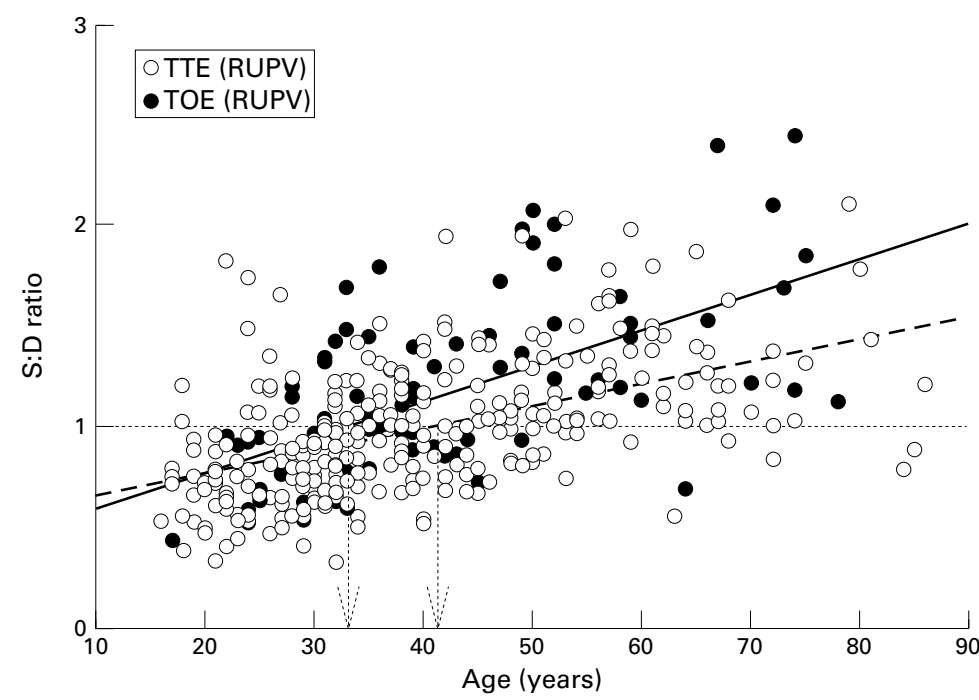

Figure 3 Relation between age and the systolic to diastolic peak flow velocity ratio (S:D). There was a significant increase in $S: D$ with advancing age in both transthoracic and transoesophageal echocardiography. However, this increase was less pronounced in transthoracic echocardiography. The dotted arrows indicate the age at which the regression lines exceed $S: D=1$ (that is, 41 years in transthoracic and 32 years in transoesophageal echocardiography). Note that there is an abundant scatter of normal $S: D$ values in all age groups. LUPV, left upper pulmonary vein; RUPV, right upper pulmonary vein; TOE, transoesophageal echocardiography; TTE, transthoracic echocardiography.

Table 3 Comparison betweeen right and left upper pulmonary veins

\begin{tabular}{llll}
\hline & LUPV $(n=72)$ & RUPV $(n=72)$ & p Value \\
\hline Peak S (cm/s) & $0.56(0.15)$ & $0.57(0.18)$ & NS \\
Peak D (cm/s) & $0.46(0.18)$ & $0.49(0.17)$ & NS \\
Peak A (cm/s) & $0.19(0.06)$ & $0.20(0.09)$ & NS \\
Sint (m) & $0.148(0.048)$ & $0.130(0.057)$ & NS \\
Dint (m) & $0.093(0.035)$ & $0.098(0.042)$ & NS \\
Aint (m) & $0.013(0.006)$ & $0.015(0.009)$ & NS \\
S decel (ms) & $248(99)$ & $203(75)$ & 0.005 \\
Ddecel (ms) & $159(75)$ & $150(56)$ & NS \\
R-S time (ms) & $230(58)$ & $156(46)$ & $<0.0001$ \\
R-D time (ms) & $511(68)$ & $544(53)$ & NS \\
Adur (ms) & $79(23)$ & $87(25)$ & NS \\
A-R' time (ms) & $58(45)$ & $70(34)$ & NS \\
S:D & $1.29(0.36)$ & $1.29(0.58)$ & NS \\
Sint/Dint & $1.73(0.64)$ & $1.54(0.90)$ & \\
\hline
\end{tabular}

Values are mean (SD).

For key to abbreviations see table 2 .

terms of any velocity or velocity-time integral between right and left upper pulmonary veins.

\section{Discussion}

This study in normal individuals generally confirms the well known age dependency of the systolic to diastolic pulmonary vein forward flow, which reflects both an increase in systolic flow and a decrease in diastolic flow. However, the $S: D$ increase seems to occur later when assessed by transthoracic echocardiography than with transoesophageal echocardiography. There is also a tendency to overestimate the backward flow duration in the pulmonary vein using transthoracic echocardiography when compared with transoesophageal echocardiography.

DIFFERENCES BETWEEN TRANSTHORACIC AND TRANSOESOPHAGEAL ECHOCARDIOGRAPHY

There is general agreement that transoesophageal echocardiography is superior to transthoracic echocardiography for Doppler PVFV measurements. ${ }^{12}$ The reproducibility and feasibility of the Doppler flow measurements, the signal to noise ratio, control over flow to ultra- sound beam angle, and control over sampling depth within the pulmonary vein are superior in transoesophageal echocardiography. The signal to noise ratio is important for the assessment of both flow velocities and flow durations. The tendency to overestimate the A wave duration using transthoracic echocardiography may be the result of less adequate signal quality or because of misinterpretation of the beginning and end of the Doppler flow signal. With low velocities - such as the A wave velocity-the signal to noise ratio in transthoracic echocardiography is additionally impaired by Doppler wall motion signals from the atrial wall. Thus the origin of reversed Doppler signals at end diastole is sometimes uncertain. This may explain the observed differences in normal values as well as the missing correlation with age in transthoracic echocardiography. The different angle between flow direction and Doppler beam direction between transthoracic and transoesophageal echocardiography may have repercussions on flow velocity measurements. In our study, however, there was no systematic tendency either to overestimate or to underestimate flow velocities using transthoracic echocardiography. The fact that peak $\mathrm{A}$ velocity and Aint were correlated with age in our transthoracic measurements may be explained by the large group size, which was sufficient to reveal weak correlations. Using transoesophageal echocardiography, the depth of the sample volume within the pulmonary vein is more easily controlled. Castello and colleagues reported that with increasing sampling depth within the right upper pulmonary vein, the $S: D$ ratio increases but the number of well defined tracings decreases. ${ }^{12}$ In relation to our present study, this suggests that in order to obtain a good Doppler trace the sample volumes may have been placed somewhat closer to the atrial orifice using transthoracic echocardiography than with transoesophageal echocardiography.

However, the differences between transthoracic and transoesophageal echocardiography may not solely be due to technical aspects, in other words to "false" differences caused by different assessment procedures. Alterations of the haemodynamic conditions induced by midazolam, as well as the stress accompanying transoesophageal probe manipulation, may cause "true" - that is physiological-changes in the PVFV profile. Prolongation of the deceleration time of early transmitral filling and the isovolumetric relaxation time, with reduction in the time-velocity integral of transmitral flow, during transoesophageal echocardiography indicate a preload reduction that may have been caused by midazolam. ${ }^{13}$ Although experimental volume loading has been shown to increase both S:D and Sint/Dint, ${ }^{14}$ midazolam-induced preload reductions may not necessarily produce the opposite effect during transoesophageal echocardiography. Acceleration of heart rate has been described as inducing an upward shift of the relation between preload and relative systolic flow rate, ${ }^{15}$ thus producing higher S:D or Sint/Dint ratios, despite a constant or even decreasing preload. This 
effect may have predominated over the pure preload effect and have led to higher S:D and Sint/Dint values during transoesophageal echocardiography than during transthoracic echocardiography. Furthermore, systolic aortic pressure has been found to be an additional independent determinant of S:D. ${ }^{14}$ In our study, systolic arterial pressure was generally lower in transoesophageal than in transthoracic echocardiography.

IMPLICATIONS FOR THE ROLE OF THE PVFV PATTERN IN DIAGNOSTIC PROCEDURES The normal range of PVFV parameters is wide. This constitutes a limitation for all diagnostic methods using the PVFV pattern. The role of the pulmonary veins for the non-invasive estimation of left ventricular end diastolic pressure has been assessed in several studies. ${ }^{516}{ }^{17}$ Rossvoll and Hatle have reported that a duration of reversed pulmonary venous flow at atrial contraction exceeding the mitral A wave duration, or a greatly reduced S:D ratio, indicated increased left ventricular end diastolic pressure. ${ }^{5}$ The relatively high scatter in these correlations, however, suggests the presence of confounding factors such as heart rate and left ventricular systolic function. ${ }^{14}{ }^{15}$ Similar problems are encountered in Doppler echocardiographic methods using the PVFV pattern for the assessment of mitral regurgitation. The depression or even reversal of systolic pulmonary venous flow has been linked qualitatively and quantitatively to mitral regurgitation. ${ }^{2}{ }^{3}$ The pronounced age dependency of the systolic peak velocity and the different age dependent behaviour of the S:D ratio between transthoracic and transoesophageal echocardiography are major confounding factors. Our present data show that there is a pronounced heterogeneity of PVFV patterns in normal individuals, thus increasing the difficulty of discerning abnormal from normal flow patterns.

\section{STUDY LIMITATIONS}

Unlike the comparisons between the right and left upper pulmonary veins, the comparisons between transthoracic and transoesophageal echocardiography were performed between different populations.

Respiration was not displayed graphically in most of the echocardiographic procedures. Therefore calculation of interobserver reproducibility includes the physiological variability caused by respiration. Furthermore, measurements were performed from long PVFV Doppler video sequences. As both observers had to make an independent choice of the best signal to be assessed, the measurements were not necessarily performed on the same PVFV
Doppler signal. Thus data on reproducibility can be considered conservative, but should closely reflect the reproducibility of the two distinct echocardiographic procedures.

\section{CONCLUSIONS}

The assessment of the PVFV pattern can be performed with good feasibility and reproducibility. However, normal values show a wide range, and they must be considered differently in transthoracic and transoesophageal echocardiography because of technical and haemodynamic differences encountered during the two imaging modes.

1 Klein AL, Tajik AJ. Doppler assessment of pulmonary venous flow in healthy subjects and in patients with heart disease. I Am Soc Echocardiogr 1991;4:379-92.

2 Klein AL, Obarski TP, Stewart WJ, et al. Transesophageal Doppler echocardiography of pulmonary venous flow: a new marker of mitral regurgitation severity. $\mathcal{F} \mathrm{Am}$ Coll Cardiol 1991;18:518-26.

3 Seiler C, Aeschbacher BC, Meier B. Quantitation of mitral regurgitation using the systolic/diastolic pulmonary venous flow velocity ratio. F Am Coll Cardiol 1998;31:1383-90.

4 Nishimura RA, Abel MD, Hatle LK, et al. Relation of pulmonary vein to mitral flow velocities by transesophageal Doppler echocardiography. Effect of different loading conditions. Circulation 1990;81:1488-97.

5 Rossvoll O, Hatle LK. Pulmonary venous flow velocities recorded by transthoracic Doppler ultrasound: relation to recorded by transthoracic Doppler ultrasound: relation to 21:1687-96.

6 Nagueh SF, Middleton KJ, Kopelen HA, et al. Doppler tissue imaging: a noninvasive technique for evaluation of left ventricular relaxation and estimation of filling pressures. $\mathcal{F}$ Am Coll Cardiol 1997;30:1527-33.

7 Oki T, Tabata T, Yamada H, et al. Clinical application of pulsed Doppler tissue imaging for assessing abnormal left ventricular relaxation. Am $\mathcal{F}$ Cardiol 1997;79:921-8.

8 Brun P, Tribouilloy C, Duval AM, et al. Left ventricular flow propagation during early filling is related to wall relaxation: a color M-mode Doppler analysis. 7 Am Coll Cardiol 1992; 20:420-32.

9 Gentile F, Mantero A, Lippolis A, et al. Pulmonary venous flow velocity patterns in 143 normal subjects aged 20 to 80 years old. An echo 2D colour Doppler cooperative study. Eur Heart f 1997;18:148-64.

10 Devereux RB, Lutas EM, Casale PN, et al. Standardization of M-mode echocardiographic left ventricular anatomic measurements. 7 Am Coll Cardiol 1984;4:1222-30.

11 Jensen JL, Williams FE, Beilby BJ, et al. Feasibility of obtaining pulmonary venous flow velocity in cardiac patients using transthoracic pulsed wave Doppler technique. F Am Soc Echocardiogr 1997;10:60-6.

12 Castello R, Pearson AC, Lenzen P, et al. Evaluation of pulmonary venous flow by transesophageal echocardiography in subjects with a normal heart: comparison with transthoracic echocardiography. F Am Coll Cardiol 1991;18:65-71.

13 Seiler C, de Marchi SF, Heule K, et al. Doppler-diastologic carnival: false and correct unmasking of disturbed left ventricular relaxation in individuals with normal transmitral flow velocity pattern [abstract]. Eur Heart $\mathcal{f}^{1998 ;}$ 19(suppl):548.

14 Hoit BD, Shao Y, Gabel M, et al. Influence of loading conditions and contractile state on pulmonary venous flow. Validation of Doppler velocimetry. Circulation 1992;86: 651-9.

15 Steen T, Voss BM, Smiseth OA. Influence of heart rate and left atrial pressure on pulmonary venous flow pattern in dogs. Am f Physiol 1994;266:H2296-302.

6 Appleton CP, Galloway JM, Gonzalez MS, et al. Estimation of left ventricular filling pressures using two-dimensional and Doppler echocardiography in adult patients with cardiac disease. Additional value of analyzing left atrial size, left atrial ejection fraction and the difference in duration of pulmonary venous and mitral flow velocity at atrial contraction. $\mathcal{F}$ Am Coll Cardiol 1993;22:197-82

17 Nakatani S, Yoshitomi H, Wada K, et al. Noninvasive estimation of left ventricular end-diastolic pressure using transthoracic Doppler-determined pulmonary venous atrial flow reversal. Am f Cardiol 1994;73:1017-8. 